\title{
Demo Abstract: Stardust: A Deep Learning Serving System in IoT
}

\author{
Shuochao Yao \\ University of Illinois at Urbana Champaign \\ syao9@illinois.edu \\ Jinyang Li \\ University of Illinois at Urbana Champaign \\ jinyang7@illinois.edu
}

\author{
Tianshi Wang \\ University of Illinois at Urbana Champaign \\ tianshi3@illinois.edu \\ Tarek Abdelzaher \\ University of Illinois at Urbana Champaign \\ zaher@illinois.edu
}

\begin{abstract}
The deep neural network becomes an increasingly crucial component in recent intelligent applications. The excessive resource consumptions of state-of-the-art neural networks, however, remains a huge impediment towards their widespread deployment in the Internet of Things (IoT). In this paper, we propose an IoT-oriented deep learning serving system, Stardust, that accelerates the neural network inference to improve the quality of IoT services. Stardust integrates several joint contributions from both the system and AI perspectives, including system performance predictor, model compression, and compressive offloading. On one hand, the performance predictor profiles and predicts the runtime characteristics of neural network operations on a particular device with the targeted runtime environment, which enables a hardware and software oriented performance optimization during model compression and offloading. On the other hand, the model compression minimizes the computation time of neural networks on different devices, and the compressive offloading diminishes the network data transferring time during the mobile-edge offloading. Moreover, all these optimizations can be done with almost no compromise on inference accuracy. The integration of these modules, therefore, collaboratively reduce the end-to-end latency of serving deep learning services that reside across embedded/mobile devices and edge servers. We deploy illustrative applications on Stardust, performing human perception tasks with on-device camera microphone and motion sensors to demonstrate the capability of Stardust serving system.
\end{abstract}

\section{CCS CONCEPTS}

- Networks; • Human-centered computing $\rightarrow$ Ubiquitous and mobile computing; • Computing methodologies $\rightarrow$ Artificial intelligence; Machine learning; • Computer systems organization $\rightarrow$ Embedded and cyber-physical systems;

\section{KEYWORDS}

Deep Learning, IoT, Offloading, Model Compression

Permission to make digital or hard copies of all or part of this work for personal or classroom use is granted without fee provided that copies are not made or distributed for profit or commercial advantage and that copies bear this notice and the full citation on the first page. Copyrights for components of this work owned by others than ACM must be honored. Abstracting with credit is permitted. To copy otherwise, or republish, to post on servers or to redistribute to lists, requires prior specific permission and/or a fee. Request permissions from permissions@acm.org.

SenSys '19, November 10-13, 2019, New York, NY, USA

(c) 2019 Association for Computing Machinery.

ACM ISBN 978-1-4503-6950-3/19/11 . \$ \$15.00

https://doi.org/10.1145/3356250.3361962
ACM Reference Format:

Shuochao Yao, Tianshi Wang, Jinyang Li, and Tarek Abdelzaher. 2019. Demo Abstract: Stardust: A Deep Learning Serving System in IoT. In The 17th ACM Conference on Embedded Networked Sensor Systems (SenSys '19), November 10-13, 2019, New York, NY, USA. ACM, New York, NY, USA, 2 pages. https: //doi.org/10.1145/3356250.3361962

\section{INTRODUCTION}

The embedded and mobile devices with the increasing sensing computation and communication capabilities are going to revolutionize the competences of daily smart physical things, performing complex sensing, recognition, and interaction tasks in an effective and efficient manner. With the recent advances of deep learning research, neural networks are becoming the state-of-the-art solutions in various application domains, including computer vision [1], speech [2], and sensing [3] tasks. Applying neural networks to IoT devices could thus bring out a new generation of services to support a more comprehensive understanding of physical and human-perception data in our daily lives [4].

However, the high execution time and energy consumption remain the major barrier to the widespread deployment of deep learning based services on mobile and embedded devices. Minimizing the execution time of neural network is critical to preserve the real-time properties and improve the quality of such IoT services as image and speech recognitions on our daily smart things. Recently, a collection of progresses have been made on improving the efficiencies of deep learning services on IoT devices, whose contributions lie in the perspectives of both system and AI. Instead of treating neural networks as black-box models used for inference, these works partly open the neural network designs in an application-agnostic way. With such designs, understanding and improving the system performance of deep learning models with a better accuracy-efficiency tradeoff becomes possible.

Previous works have contributed separately to related areas, including effectively modelling sensing signals [5], system performance predictor [6] and deep learning structure compression [7]. In this paper, we integrate previous contributions with our recent designs on compressive offloading, presenting a joint designs for IoT deep learning serving system, called Stardust. Stardust organizes these technical components in a coherent manner so that they can mutually enhance each other and reduce the end-to-end serving latency of deep learning based IoT services collaboratively.

We demonstrate the efficacy of Stardust system based on traditional recognition tasks with multi-sensor fusion and enhancement. The application provides traditional visual and speech recognition services based on on-board camera microphone and motion sensors. Stardust is operated on a general embedded-edge system for serving low-latency inference. 


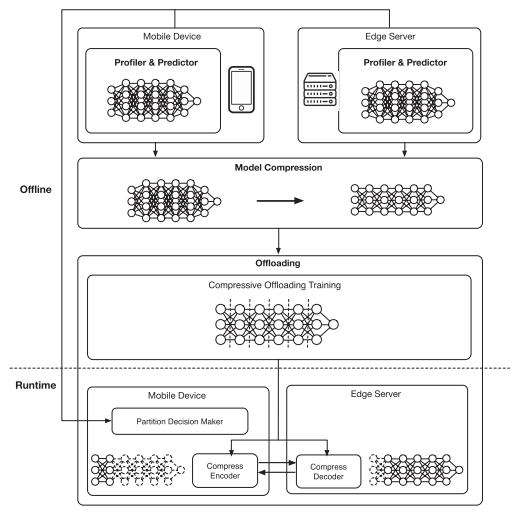

Figure 1: The overview of Stardust system.

\section{STARDUST DESIGNS}

The overview design of Stardust is illustrated in Figure 1, where the deployment includes two phases: offline profiling \& training and runtime management.

\subsection{Offline Profiling \& Training}

The designing principles of Stardust are twofold. On one hand, Stardust optimizes the neural network by understanding its system behaviours in the targeted runtime environment. On the other hand, Stardust improves the efficiency-accuracy tradeoff by partly opening the black-box neural network models but in an applicationagnostic manner. Therefore, there are three main components in this subsection. How to understand and predict the system behaviour of neural network operations in the targeted runtime environment? How to reduce the execution time of neural network with a minimal impact on prediction performance? How to reduce the network transmission time of neural network with a minimal impact on prediction performance?

2.1.1 Performance Predictor. Stardust adopts the profiling based operation-level performance predictor proposed in FastDeepIoT [6] During the offline phase, Stardust first profiles the execution time of various neural network operations (e.g., convolution, recurrent, fully connected) with different configurations on embedded devices and edge server respectively. Then Stardust trains a tree-structure linear regression model [6] based on the profiled data, which is served as the performance predictor to predict the execution time of neural network operations during runtime. The performance predictors on the embedded and the edge serve as the optimization guidelines for other components.

2.1.2 Structure Compression. In order to reduce the execution time of neural network on either the embedded or the edge, Stardust compresses the structure of neural network by finding the minimum number of non-redundant hidden elements, such as filters and dimensions required by each layer, while keeping the performance of sensing applications the same [7]. The structure compression usually achieve better system efficiency than the unstructured compression. Moreover, the slim neural network can be tailored to the runtime environment of the targeted devices, where Stardust uses performance predictors to guide the structure compression process that fit best to the particular system.

2.1.3 Compressive offloading. Even with the compressed model, embedded devices may not be powerful enough to execute a neural network within the timing requirement. Edge offloading, therefore, becomes a promising choice for reducing the end-to-end inference latency. However, the network data transmission time takes up most of the time during offloading, which becomes the bottleneck of offloading. Stardust designs the compressive offloading that can significantly reduce the data transmission time by compressing the size of data on an embedded device and reconstructing the data on the edge server. The compressive encoder and decoder are two trainable neural networks which can recover the compressed data with almost no compromise on the prediction accuracy.

\subsection{Runtime}

The runtime of Stardust is a scheduling module that decides the offloading pointing for the deep learning service. Based on the performance predictor on the embedded and the edge as well as the current network condition, runtime scheduler can decide the best offloading point that minimizes the end-to-end inference latency. By defining the objective of scheduler with the corresponding performance predictor, Stardust is able to schedule deep learning service based on deadline or minimizing embedded energy consumption.

\section{DEMONSTRATION}

We prototype Stardust with a Raspberry Pi as the embedded device and Jetson AGX Xavier as the edge server. Vision and speech recognition servers are supported with on device camera and microphone as well as motion sensors for sensing enhancement. System profiling, model compression and training compressive encoder \& decoder are finished beforehand. Stardust is operated to minimizing the end-to-end inference time during the demonstration.

\section{ACKNOWLEDGEMENT}

Research reported in this paper was sponsored in part by NSF under grants CNS 16-18627 and CNS 13-20209 and in part by the Army Research Laboratory under Cooperative Agreements W911NF-09-20053 and W911NF-17-2-0196. The views and conclusions contained in this document are those of the authors and should not be interpreted as representing the official policies, either expressed or implied, of the Army Research Laboratory, NSF, or the U.S. Government. The U.S. Government is authorized to reproduce and distribute reprints for Government purposes notwithstanding any copyright notation here on.

\section{REFERENCES}

[1] Kaiming He, Xiangyu Zhang, Shaoqing Ren, and Jian Sun. Deep residual learning for image recognition. In Proceedings of the IEEE conference on computer vision and pattern recognition, pages 770-778, 2016

[2] Awni Hannun, Carl Case, Jared Casper, Bryan Catanzaro, Greg Diamos, Erich Elsen, Ryan Prenger, Sanjeev Satheesh, Shubho Sengupta, Adam Coates, et al. Deep speech: Scaling up end-to-end speech recognition. arXiv preprint arXiv:1412.5567.

[3] Shuochao Yao, Shaohan Hu, Yiran Zhao, Aston Zhang, and Tarek Abdelzaher. Deepsense: A unified deep learning framework for time-series mobile sensing data processing. In Proceedings of the 26th International Conference on World Wide Web. International World Wide Web Conferences Steering Committee, 2017.

[4] Shuochao Yao, Yiran Zhao, Aston Zhang, Shaohan Hu, Huajie Shao, Chao Zhang, $\mathrm{Lu} \mathrm{Su}$, and Tarek Abdelzaher. Deep learning for the internet of things. Computer, 51(5):32-41, 2018.

[5] Shuochao Yao, Ailing Piao, Wenjun Jiang, Yiran Zhao, Huajie Shao, Shengzhong Liu, Dongxin Liu, Jinyang Li, Tianshi Wang, Shaohan Hu, et al. Stfnets: Learning sensing signals from the time-frequency perspective with short-time fourier neural networks. In The World Wide Web Conference. ACM, 2019.

[6] Shuochao Yao, Yiran Zhao, Huajie Shao, ShengZhong Liu, Dongxin Liu, Lu Su, and Tarek Abdelzaher. Fastdeepiot: Towards understanding and optimizing neural network execution time on mobile and embedded devices. In Proceedings of the 16th ACM Conference on Embedded Networked Sensor Systems. ACM, 2018.

[7] Shuochao Yao, Yiran Zhao, Aston Zhang, Lu Su, and Tarek Abdelzaher. Deepiot: Compressing deep neural network structures for sensing systems with a compressor-critic framework. In Proceedings of the 15th ACM Conference on Embedded Network Sensor Systems, page 4. ACM, 2017. 